\title{
tdkp/AQUA: Unified modeling of electroluminescence in nanostructures
}

\section{Journal Article}

Author(s):

Steiger, Sebastian; Veprek, Ratko G.; Witzigmann, Bernd

Publication date:

2009

Permanent link:

https://doi.org/10.3929/ethz-b-000422929

Rights / license:

In Copyright - Non-Commercial Use Permitted

Originally published in:

Optical and Quantum Electronics 41(7), https://doi.org/10.1007/s11082-010-9360-8 


\title{
tdkp/AQUA: Unified modeling of electroluminescence in nanostructures
}

\author{
Sebastian Steiger • Ratko G. Veprek • \\ Bernd Witzigmann
}

Received: 29 August 2009 / Accepted: 7 January 2010 / Published online: 12 February 2010

C) Springer Science+Business Media, LLC. 2010

\begin{abstract}
This article summarizes the capabilities of the optoelectronic simulation framework $t d k p / A Q U A$ aimed at the description of electroluminescence in semiconductor nanostructures such as light-emitting diodes. $t d k p$ is a stand-alone finite-element software able to accurately calculate strain, built-in fields due to spontaneous and piezoelectric polarization, bound quantum states using $\mathbf{k} \cdot \mathbf{p}$ theory, gain and luminescence spectra in zero- to three-dimensional structures. AQUA calculates transport through nanostructures using a model which accounts for the distinct behaviour of carriers confined to active regions and unconfined carriers. Furthermore, it computes electroluminescence spectra via a self-consistent coupling of the confined carriers to quantum-mechanical calculations using $t d k p$. Two examples are presented which highlight the versatility and generality of the developed framework.
\end{abstract}

Keywords Device simulation $\cdot$ LED $\cdot$ Electroluminescence $\cdot \mathrm{kp}$ theory $\cdot$ Drift-diffusion

\section{Introduction}

Light-emitting diodes (LED) have become one of the great hopes of the beginning 21 st century. Their longevity, superior energy efficiency and impeccable color characteristics destines them as replacements in most lighting applications, with potential energy savings equivalent to a gigaton of $\mathrm{CO}_{2}$ per year (Schubert et al. 2006). In this respect it is highly desirable

\footnotetext{
S. Steiger $(\varangle) \cdot$ R. G. Veprek

Integrated Systems Laboratory, ETH Zurich, Gloriastr 35, 8092 Zurich, Switzerland

e-mail: steiger@iis.ee.ethz.ch; steigers@phys.ethz.ch

Present Address:

S. Steiger

Winterthurerstr 296, 8057, Zurich, Switzerland

B. Witzigmann

Computational Electronics and Photonics, University of Kassel, 34121, Kassel, Germany

e-mail: bernd.witzigmann@uni-kassel.de
} 
to precisely understand the physical processes governing current devices as well as explore novel LED concepts.

The complexity of the involved physics as well as the often three-dimensional spatial dependence of key quantities usually limits the feasibility of analytical models. $t d k p / A Q U A$ is a numerical simulation framework developed by the authors which aims at the comprehensive, self-consistent description of the essential processes governing nano-LED operation. Key to this task is the description of the active, light-emitting region, which exhibits quantization effects in one, two or three dimensions. One of the central goals of $t d k p / A Q U A$ is to unify the description with respect to the dimensions of confinement and even allow for a mixture of different quantum regions in the same device.

In the following sections, an overview of the employed models is given and two application examples are briefly discussed.

\section{Model}

$2.1 \mathrm{tdkp}$ - Strain, polarization, $\mathbf{k} \cdot \mathbf{p}$ quantum states, gain and luminescence

$t d k p$ (Veprek et al. 2008) is a stand-alone software library based on a continuum description to calculate strain, spontaneous and piezoelectric polarization and its induced potential, quantum states within the multiband $\mathbf{k} \cdot \mathbf{p}$ band structure model and gain and luminescence spectra for nanostructures of arbitrary dimensionality. A flexible finite-element discretization is employed for all differential equations.

Strain calculations are based on a linear elasticity description in which the strain energy

$$
\frac{1}{2} \int d V C_{i j}\left(\epsilon_{i}+\epsilon_{i}^{0}\right)\left(\epsilon_{j}+\epsilon_{j}^{0}\right)
$$

is minimized with respect to the displacement $\mathbf{u}$, where $\epsilon_{i}$ denotes the strain $\epsilon_{i j}=$ $\frac{1}{2}\left(\frac{\partial u_{j}}{\partial x_{i}}+\frac{\partial u_{i}}{\partial x_{j}}\right)$ in Voigt notation. Equation 1 includes the energy due to intrinsic strain $\epsilon^{0}$ and is minimized together with appropriate boundary conditions, typically a combination of Neumann and Dirichlet types. The polarization is then determined by

$$
P_{i}=P_{i}^{s p}+\sum_{j} e_{i j} \epsilon_{j}+\sum_{j k} B_{i j k} \epsilon_{i j} \epsilon_{k},
$$

where $\mathbf{P}^{s p}$ is the spontaneous polarization inherent to the material and the remaining terms denote the linear and quadratic contributions of the piezoelectric polarization. The converse piezoelectric effect is not accounted for. The finite-element formulation allows for an exact inclusion of surface and volume charges, which are related to the polarization via Gauss' law. This enables an accurate modeling of material interfaces in calculations of polarizations and induced potentials.

$\mathbf{k} \cdot \mathbf{p}$ envelope function theory (Kane 1982) is particularly suitable for finding quantum states in optoelectronic structures because it accurately models the band structure around the $\Gamma$-point without overextending computational costs. Key to the robustness and reliability of such calculations is the elliptic formulation of the equations (Veprek et al. 2007). All standard $\mathbf{k} \cdot \mathbf{p}$ models for zincblende and wurtzite structures, including strain corrections within Bir-Pikus theory (Bir and Pikus 1974), are implemented.

Lastly, gain spectra are calculated for a given density using a semiclassical description (Haug and Koch 2004) in which the electromagnetic field is classical, carrier states are 


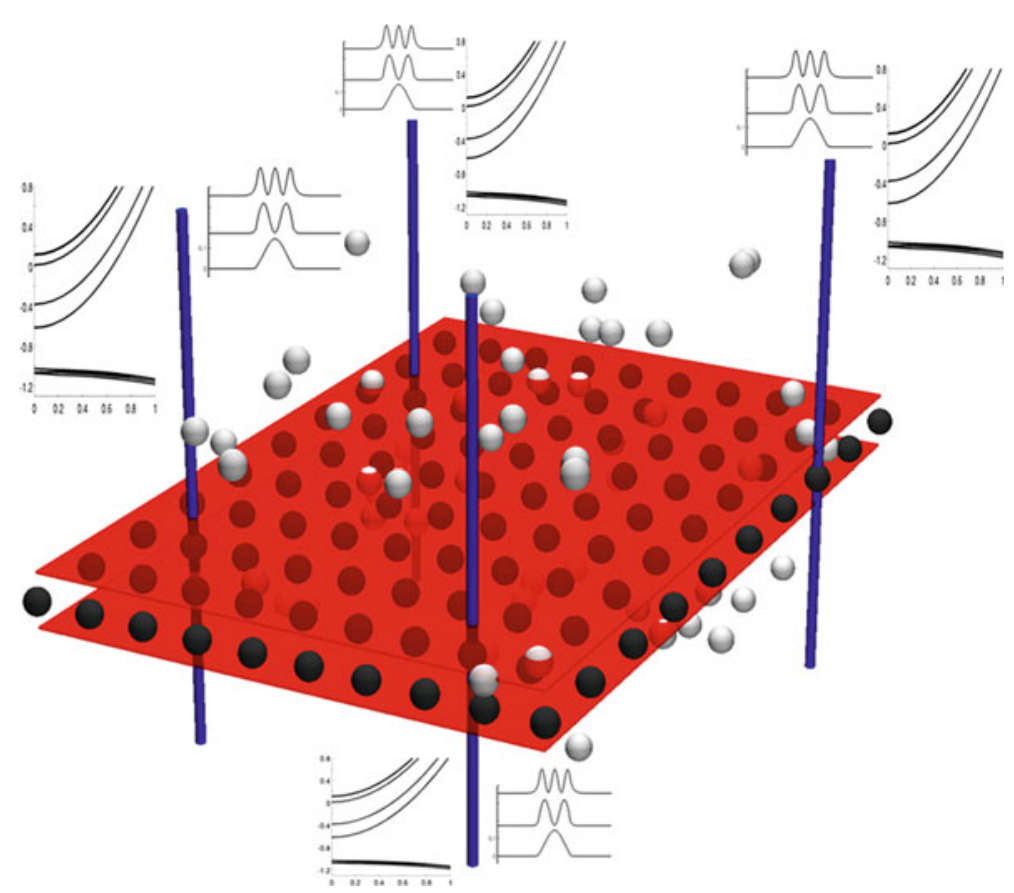

Fig. 1 AQUA transport model illustrated for a quantum-well. White/unordered: 3D unbound carriers able to move in all directions. Black/ordered: 2D carriers confined to the well. Transport only occurs in the lateral directions. Vertical bars: Slices where $\mathbf{k} \cdot \mathbf{p}$ calculations are carried out to determine the shape of the bound population in the confined direction and the magnitude of the rafdiative recombination

quantum-mechanical and energetic quasi-equilibrium within electrons and holes is assumed. Luminescence spectra are determined within the validity of the Kubo-Martin-Schwinger relation. Many-body effects such as band gap renormalization, exciton peaks and Coulomb enhancement are optionally neglected (free-carrier level) or treated on the screened Hartree-Fock level. In the latter, direct and exchange interactions are considered with a dielectric constant which is calculated from the Lindhard formula.

\subsection{AQUA - Carrier transport and self-consistency with luminescence}

AQUA (Steiger et al. 2008) models carrier transport in a semi-coherent picture in which carriers completely lose or retain coherence in a certain direction. The carrier system is partitioned into unbound populations, able to move in all directions, and bound populations confined to the active region, be it a quantum-well, -wire or -dot. Bound carriers are described by their quantum-mechanical wavefunctions in the confinement directions whereas they are able to move by drift and diffusion in the remaining directions.

Figure 1 illustrates the transport model for the case of a single quantum-well. Two populations exist, one of which is confined to the well. The shape of these carriers in the confined direction is determined by the $\mathbf{k} \cdot \mathbf{p}$ problem which is solved at a set of locations, termed $\mathbf{k} \cdot \mathbf{p}$ slices, specified by the user. These are also the locations where radiative recombination is calculated on the basis of the $\mathbf{k} \cdot \mathbf{p}$ results. The wavefunction shape and magnitude of the luminescence between the slices is interpolated. 
The densities of the different electron populations are hence determined by continuity equations

$$
\begin{aligned}
& \nabla \cdot \mu_{n} n_{3} \nabla E_{F n, 3}=R_{n 3}-G_{n 3}, \\
& \nabla \cdot \mu_{n} n_{2} \nabla E_{F n, 2}=R_{n 2}-G_{n 2},
\end{aligned}
$$

where $E_{F n, d}=E_{c}-e \phi-\frac{d}{2} \log m_{e}+k T \log n$ is the quasi-Fermilevel of a $d$-dimensional electron population. The amount of continuity equations solved depends on the number of wells, wires and dots in the nanostructure. Coupling of the populations happens through capture, acting as recombination $\left(R_{d}\right)$ and generation $\left(G_{d-1}\right)$ terms in the continuity equations, and the common electrostatic potential $\phi$, governed by the Poisson equation

$$
\nabla \cdot(\epsilon \nabla \phi)=e\left(n^{(t o t)}-p^{(t o t)}-N_{d}\right) .
$$

Here $N_{d}$ is the net ionized doping density and the densities are the sum of bound and unbound populations.

Transport equations are discretized using the Scharfetter-Gummel finite volume method and solved by Newton iteration using analytic differentiation with an automated chain rule. The approach of our implementation towards the Newton solution and the general structure allows for an easy exchange or extension of the employed models. In fact, the introduction of different meshes on which the different populations exist necessitates a multitude of interpolations between these grid. Without an automated dependency and differentiation scheme, this plethora of equations would be intractable. Radiative recombination of bound carriers as well as their shape in the quantized direction(s), influencing the Poisson equation, is calculated using $t d k p$. A predictor-corrector scheme is employed to facilitate self-consistence.

At the core of $t d k p / A Q U A$ lie a finite-element solver and a Newton solver. Top-level program steering is done using the scripting language python into which the $t d k p$ and $A Q U A$ libraries are imported. The two libraries together comprise more than 100,000 lines of C++ code, with OpenMP parallelization of the most time-consuming sections. $t d k p / A Q U A$ depends on non-commercial third-party software only. Input and output is done in the DF-ISE format for which a small read-write library called sebise is provided. Experimental support for the open-source visualization toolkit VTK is also provided. Several linear solvers can be employed, among which the sequential, direct solver UMFPACK, the parallel, direct solver PARDISO and the parallel, direct or iterative solver PETSC. For the eigenvalue solutions $A R P A C K$ is employed together with a shift-and-invert strategy.

\section{Application examples}

\subsection{Nanocolumn LED}

The InGaN/GaN nanocolumn LED (Kishino et al. 2007; Boecklin et al. 2010) depicted in Fig. 2 has a three-dimensional dependence of many of the aforementioned quantities. The finite extension in lateral directions allows for strain relaxation towards the surface. There is a fragile interplay between the strain-induced lowering of the bulk band gap towards the periphery and a counter-acting increase due to a smaller polarization potential. 3D calculations of states bound to the quantum disk show that their location strongly depends on the assumed parameter sets. Using 50\% of the polarization parameters in Piprek (2007), the resulting band edges are rather flat on the inside but drop sharply towards the column surface. 

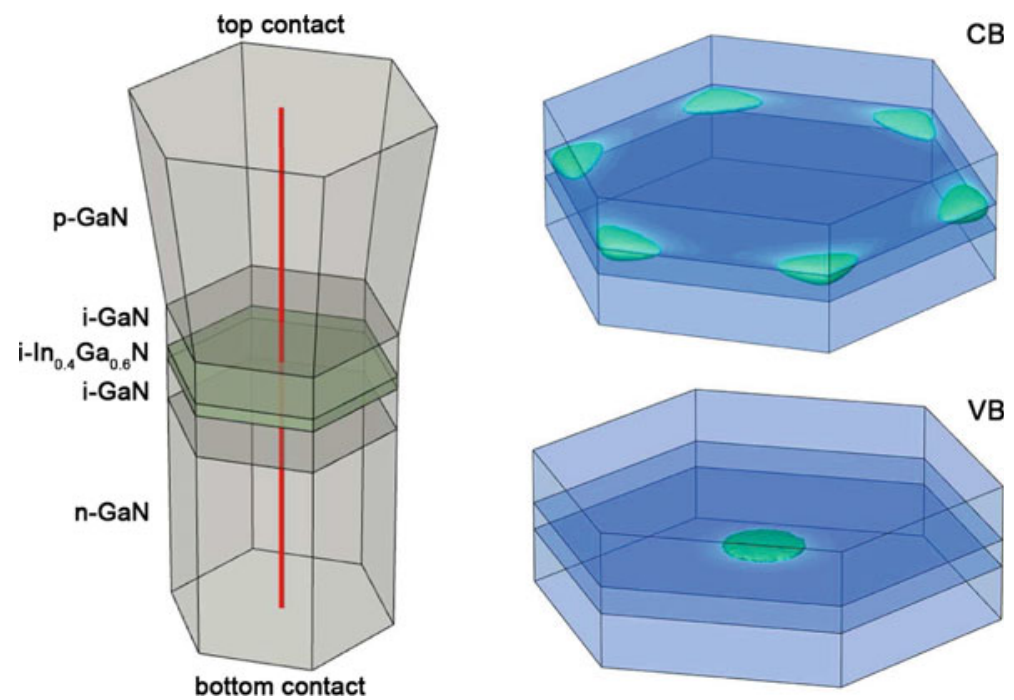

Fig. 2 Nanocolumn LED structure and first bound 3D electron and hole state including strain and polarization effects
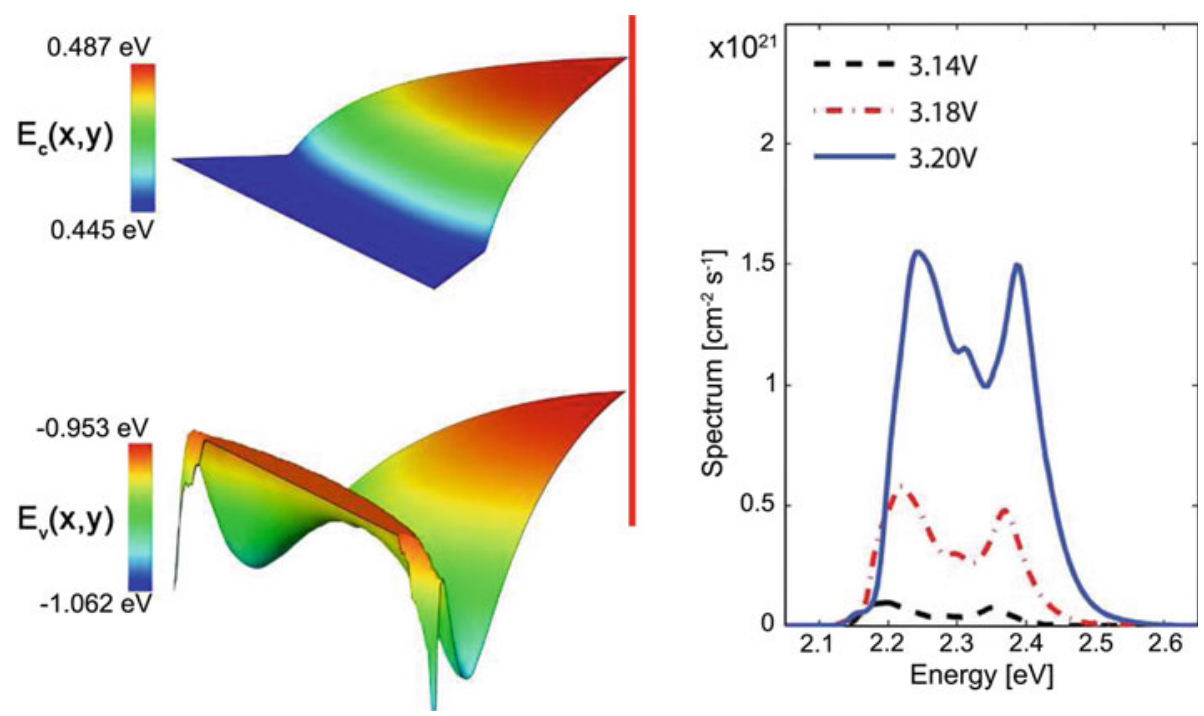

Fig. 3 Nanocolumn LED lateral drift-diffusion potential for bound carriers (only a sixth of the column is shown) and emission spectra

The density of states (not shown) exhibits no clear energetic separation between the states, and an analysis of the overlap integrals shows a very complicated mixture between bright and dark transitions. However, it is important to observe that high scattering renders the picture of coherent 3D states inadequate. Therefore, a quantum-well picture is adopted for the carrier transport where lateral drift-diffusion movement happens in a potential corrected near the surface (Fig. 3). 

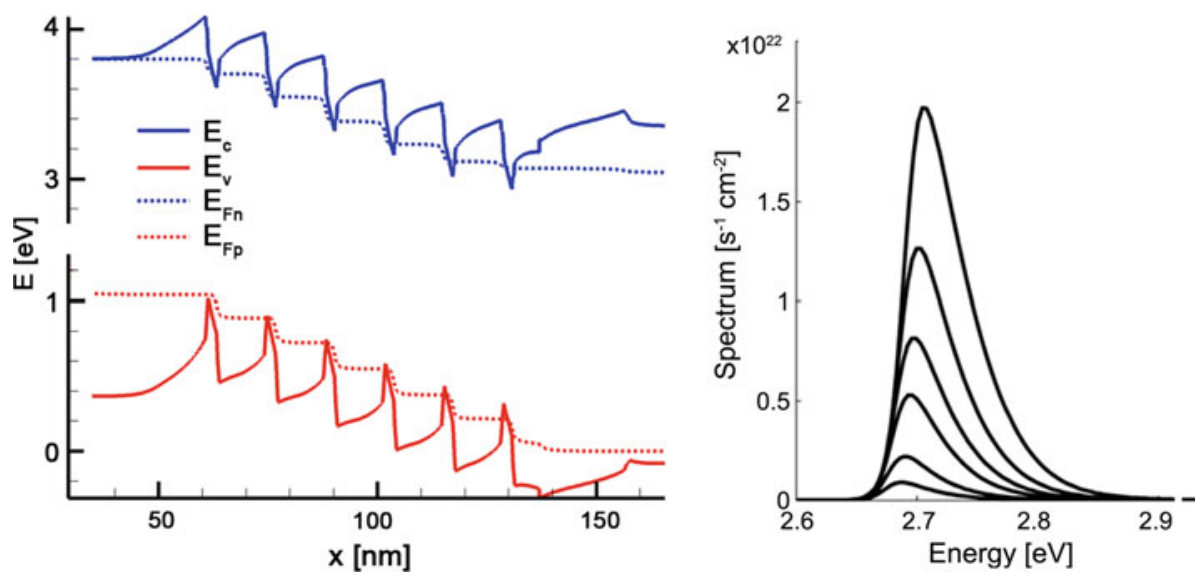

Fig. 4 InGaN/GaN multi-quantum-well LED band edges, quasi-Fermilevels and emission spectra near turnon
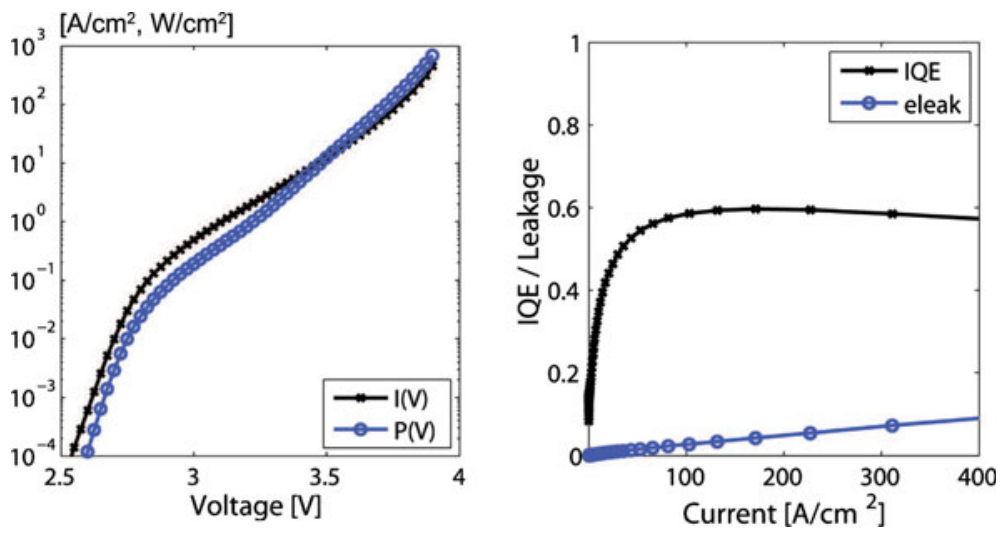

Fig. $5 I-V$ and $P-V$ curves, internal quantum efficiency and electron leakage of the InGaN MQW LED

The resulting luminescence (Fig. 3) arises near the column surface. Comparing the device characteristics to a planar device with similar materials, the spectra are red-shifted and significantly broader. Furthermore, the turn-on voltage is lower than in planar devices because of the reduced polarization barrier near the surface. So far, comparsion to experiment was hindered by large uncertainties about experimental material compositions and shapes. With the advent of the deterministic growth of uniform nanocolumn arrays (Kishino et al. 2009), such comparisons will be facilitated.

\subsection{Nitride-based MQW-LED}

A second example is the planar InGaN/GaN multi-quantum-well LED shown in Fig. 4 which resembles current high-power commercial LEDs (Kim et al. 2007). A central matter in these devices is the efficiency droop at high output power (Fig. 5) which may have several physical origins. Apart from temperature effects, $t d k p / A Q U A$ is able to describe these mechanisms and provide insight into the importance of electron leakage and Auger recombination. 
The relative importance of the effects again strongly depends on parameters whose correctness is not well-established. They include the polarization coefficients (see Eq. 2), the Auger coefficients, and carrier capture times into the quantum-wells. In the present example, the efficiency droop seems to be caused by electron leakage despite an electron blocking layer. It must be noted that the model breaks down at high currents due to the neglected temperature effects.

\section{Conclusion}

The device simulator $t d k p / A Q U A$ allows for a comprehensive investigation of current and future semiconductor LEDs. Nevertheless, a lot of future improvements can be envisaged to extend the capabilities. Currently no models for the external quantum efficiency exist, and the light-matter coupling is calculated assuming free-space light modes. Such limitations can be overcome by coupling the simulator to an electromagnetic solver. Furthermore, optical generation and nonuniform temperatures could be investigated using appropriate equations within the Newton solution framework. Lastly, no lasing model currently exists and all computations are done at steady-state.

Acknowledgments This work was funded through grant 200021-107932 of the Swiss National Science Foundation (SNSF).

\section{References}

Bir, G.L., Pikus, G.E.: Symmetry and Strain-Induced Effects in Semiconductors. Wiley, New York (1974)

Boecklin, C., Veprek, R.G., Steiger, S., Witzigmann, B.: A comprehensive microscopic anaysis of ingan/gan nanocolumn leds. submitted (2010)

Haug, H., Koch, S.W.: Quantum Theory of the Optical and Electronic Properties of Semiconductors. World Scientific, Singapore (2004)

Kane, E.O: Energy band theory. In: Paul, W. (ed.) Handbook on Semiconductors, vol. 1, pp. 194-217. North Holland, Amsterdam (1982)

Kim, M.H., Schubert, M.F., Dai, Q., Kim, J.K., Schubert, E.F., Piprek, J., Park, Y.: Origin of efficiency droop in gan-based light-emitting diodes. Appl. Phys. Lett. 91, 183-507 (2007)

Kishino, K., Kikuchi, A., Sekiguchi, H., Ishizawa, S.: Ingan/gan nanocolumn leds emitting from blue to red. Proc. SPIE 6473, 64-730T (2007)

Kishino, K., Sekiguchi, H., Kikuchi, A.: Improved ti-mask selective-are-growth (sag) by rf-plasmaassisted molecular beam epitaxy demonstrating extremely uniform gan nanocolumn arrays. J. Cryst. Growth 311, 2063-2068 (2009)

Piprek, J.: Nitride Semiconductor Devices: Principles and Simulation. Wiley, New York (2007)

Schubert, E.F., Kim, J.K., Luo, H., Xi, J.Q.: Solid-state lighting-a benevolent technology. Rep. Prog. Phys. 69, 3069-3099 (2006)

Steiger, S., Veprek, R.G., Witzigmann, B.: Unified simulation of transport and luminescence in optoelectronic nanostructures. J. Comput. Electron. 7, 509-520 (2008)

Veprek, R.G., Steiger, S., Witzigmann, B.: Ellipticity and the spurious solution problem of kp envelope equations. Phys. Rev. B 76, 165-320 (2007)

Veprek, R.G., Steiger, S., Witzigmann, B.: Reliable kp band structure calculation for nanostructures using finite elements. J. Comput. Electron. 7, 521-529 (2008) 